\title{
Morfogeometría de los peces del género Hyphessobrycon (Characiformes: Characidae), grupo heterorhabdus, en Venezuela
}

\author{
Carlos A. García-Alzate, César Román-Valencia \& Melissa I. González \\ Universidad del Quindío, Laboratorio de Ictiología, A. A. 2639, Armenia, Quindío, Colombia; \\ cagarcia@uniquindio.edu.co,ceroman@uniquindio.edu.co,meli328@gmail.com
}

Recibido 16-VIII-2009. Corregido 12-III-2010. Aceptado 19-IV-2010.

\begin{abstract}
Morphogeometry of fishes in the genus Hyphessobrycon (Characiformes: Characidae), heterorhabdus group, from Venezuela. Hyphessobrycon is the most numerous and morphologically complex genus of Characidae, that includes 122 valid species, 17 of which are present in Venezuela. Different methods have been proposed for species identification within this genus. We present a morphometric analysis for the species Hyphessobrycon, heterorhabdus group. A total number of 310 individuals were photographed and analyzed by means of Box Truss method. The combination of 16 homologous landmarks and the design of Box Truss yielded 28 measurements. The Principal Components Analysis (PCA) indicated that the differences between the species are related to the distance between snout and the supraoccipital $(81.7 \%)$, and between the supraoccipital and the base of the pectoral fin (11.87\%). All species showed allometric growth except for Hyphessobrycon tuyensis, which resulted isometric in the Box Truss I. The partial deformations describe the form differences related to the cephalic region and the body depth. The great body form similarity is related to the phylogeny and biogeography of these species. Rev. Biol. Trop. 58 (3): 801-811. Epub 2010 September 01.
\end{abstract}

Key words: allometric, box truss, partial deformations, association, tropical fish.

Hyphessobrycon es un género de pequeños peces carácidos que incluye alrededor de 122 especies válidas (García-Alzate 2009) distribuidas desde el sur de México hasta la cuenca del Río de la Plata. La definición del género fue inicialmente propuesta por Durbin (en Eigenmann 1908), reevaluada recientemente por García-Alzate (2009) y se define por la combinación de los siguientes caracteres exclusivos: tercer infraorbital no en contacto con el preopérculo, seis o siete dientes en fila interna del premaxilar, dos dientes tricúspides en la fila externa del premaxilar, dientes más anteriores del dentario (frontales) del mismo tamaño que los laterales y el proceso lateral del centrum no se extiende hasta el borde ventral posterior del basioccipital.

Se ha propuesto el reconocimiento de grupos de especies de este género basados en un patrón de coloración o pigmentación (Géry
1977, Weitzman \& Palmer 1997); de interés particular en éste trabajo es el grupo monofilético heterorhabdus, definido por la presencia de una banda lateral oscura (García-Alzate et al. 2008a) y por dos sinapomorfias: 13 a 14 epipleurales y metapterigoide sin proyección ventral, no en contacto con el simpléctico (García-Alzate 2009). Para Venezuela se reportan 17 especies de Hyphessobrycon, en las cuencas del Orinoco, Río Negro, Maracaibo y los drenajes del Caribe (García-Alzate et al. 2008b); de las cuales cinco especies pertenecen al grupo heterorhabdus: H. diancistrus Weitzman, 1977; H. sovichthys Schultz 1944; H. fernandezi (Fernández-Yépez 1972); H. paucilepis García-Alzate et al. 2008b y H. tuyensis García-Alzate et al. 2008b (Fig. 1).

La complejidad morfológica de Hyphessobrycon ha causado problemas para definir las especies, por lo cual se requieren análisis 


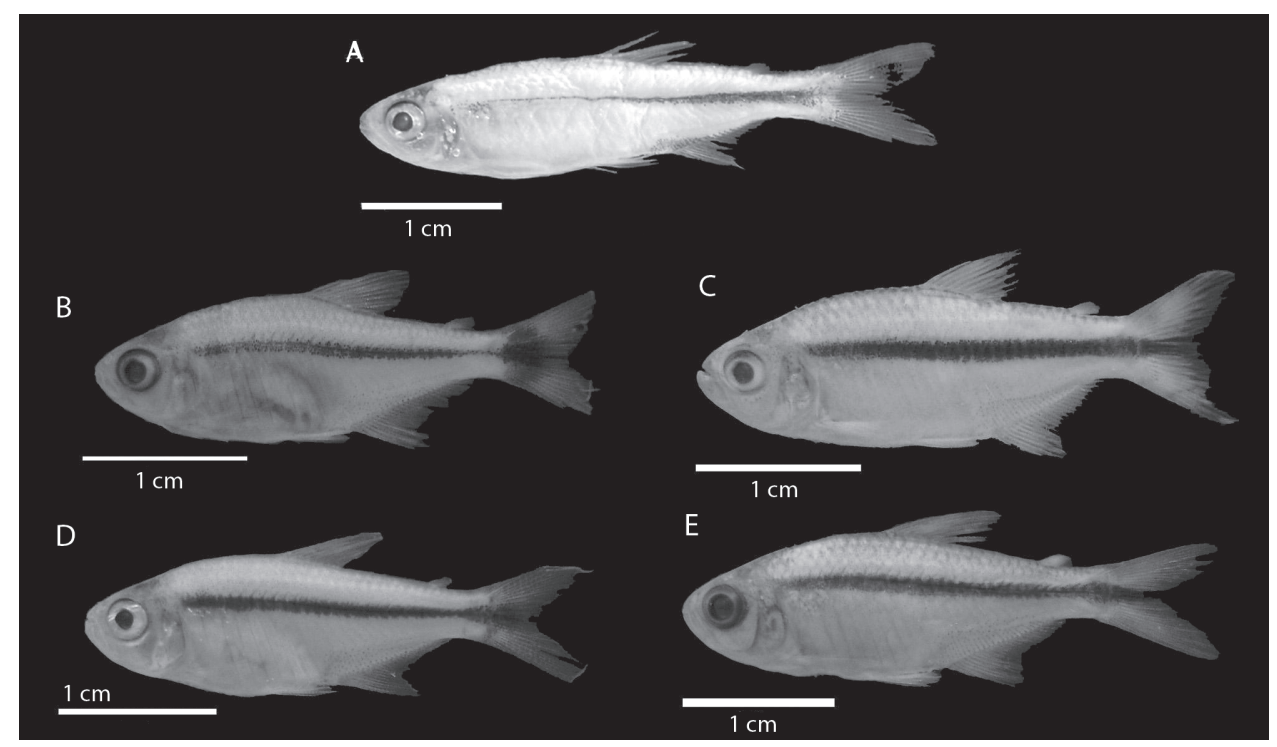

Fig. 1. Especies de Hyphessobrycon grupo heterorhabdus de Venezuela. $\mathrm{A}=H$. diancistrus, $\mathrm{B}=H$. fernandezi, $\mathrm{C}=H$. sovichthys, $\mathrm{D}=H$. paucilepis y $\mathrm{E}=H$. tuyensis.

Fig. 1. Species of Hyphessobrycon heterorhabdus-group in Venezuela. A=H. diancistrus, $\mathrm{B}=H$. fernandezi, $\mathrm{C}=H$. sovichthys, $\mathrm{D}=$ H. paucilepis and $\mathrm{E}=H$. tuyensis.

de tipo morfológico que contribuyan a diferenciar las especies relacionadas. Sobre éste propósito, la morfometría geométrica representa un recurso metodológico en la determinación de medidas discriminantes entre especies que pueden ser utilizadas a nivel taxonómico (González-García \& Lopera 2009), además, permite la distinción entre forma y talla, comparar organismos mediante sus estructuras homólogas, cuantificar la variabilidad de la forma en múltiples escalas espaciales, y estudiar las posibles correlaciones entre la variabilidad y otros parámetros morfológicos y/o ambientales (Ruiz-Calderón \& Cipriani 2006). Por lo anterior, el objetivo del estudio es realizar un análisis morfogeométrico de las especies del género Hyphessobrycon grupo heterorhabdus presentes en Venezuela.

\section{MATERIAL Y MÉTODOS}

Los ejemplares examinados pertenecen a los siguientes museos: Colombia: Laboratorio de Ictiología de la Universidad del Quindío, Armenia (IUQ), Instituto de Ciencias Naturales-Museo de Historia Natural, Universidad Nacional de Colombia, Bogotá (ICN-MHN). Venezuela: Museo de Biología, Instituto de Zoología Tropical, Universidad Central de Venezuela, Caracas (MBUCV); Museo de Historia Natural, Fundación La Salle de Ciencias Naturales, Caracas (MHNLS), Museo de Zoología, Universidad Experimental de los Llanos Occidentales "Ezequiel Zamora", Guanare (MCNG) y Colección de Peces de la Universidad Experimental Centroocidental "Lisandro Alvarado", Barquisimeto (CPU-CLA).

\section{Material Examinado}

Hyphessobrycon diancistrus: Venezuela. MBUCV-V 30835, (3) (31.40-34.15mm de LE.); Río Cataniapo, aguas abajo de la boca del caño Colorado, río abajo de San Pedro, Amazonas. MBUCV-V 30861, (10) (26.58$30.89 \mathrm{~mm}$ de LE.); caño Gavilán, en playa 
al lado izquierdo a una hora de la comunidad Gavilán, Amazonas. MBUCV-V 31944, (10) (24.90-33.62mm de LE.); boca del caño Vaquiro, afluente del río Cataniapo, Amazonas. Hyphessobrycon fernandezi: Venezuela. MBUCV 24113, (12) (22.45-31.02mm de LE.) Caño Dieguito bajo el puente vía Tocuyo, Refugio de Fauna Silvestre de Cuare, Falcón. MBUCV 24232, (2) (25.92-26.18mm de LE.) río Yaracuy (boca), Yaracuy. MCNG 14058, (47) (15.91-34.61 mm. LE), quebrada Cupa, finca de Víctor Atria, cerca de Aroa en la vía a Duana, Yaracuy. MCNG 32126, (6) (23.75-27.87mm de LE.) Caño Minapo, al norte de Palma Sola aprox. 4km., Falcón. MCNG 41736, (1) (27.40mm de LE.) Río Portuguesa, La Aduana vía a Nueva Florida, Portuguesa. MCNG 52207, (3) (24.83-31.94mm de LE.) Canal en la planta procesadora de granos en Payara, Portuguesa. MCNG 52216, (1) (23.93mm de LE.) Un caño después del canal en la vía a Payara, Portuguesa, MBUCV-V 34105, (1) Neotipo: quebrada Cupa, finca de Víctor Atria, cerca de Aroa en la vía a Duaca, $10^{\circ} 29^{\prime} 50^{\prime \prime}$ N, 6852'20" W, Yaracuy. Hyphessobrycon paucilepis: Venezuela. Holotipo: IUQ 1897, (26.77mm de LE.); embalse los Quediches, Canal del aliviadero, estado de Lara. Paratipos: MBUCV-V 23710, (3) (23.78-27.43mm de LE.) colectado con el holotipo. MCNG 36938, (1) $(23.13 \mathrm{~mm}$ LE.) Parque Nacional Aguaro Guariquito, río Aguaro, paso Garcerito. CPUCLA 532, (3) (25.70-30.47mm de LE) Ciénaga de Puricaure vía Carora El Venado, aprox. $1 \mathrm{~km}$. de Puricaure, vía quebrada arriba, Lara. MBUCV 6933, 8 (11.84-23.35mm de LE) Burere, carretera Carora-Cabinas, Lara. Hyphessobrycon sovichthys: Colombia. ICNMHN 2360, (5) (27.26-30.09mm de LE.) Pozo anóxico aislado del río Catatumbo, aguas abajo corregimiento la Gabarra, río la Gabarra cuenca del río Catatumbo, Norte de Santander. Venezuela. MBUCV 29776, (10) (17.70-28.95mm de LE.) caño la Guardia, balneario abajo del puente, carretera Casigua-Maracaibo, Zulia. MCNG 19696, (21) (18.93-28.96mm de LE.). MCNG 24833, (1) (27.28mm LE.). MCNG 24912, (7) $(27.67-29.90 \mathrm{~mm}$ de LE.) cañito afluente del río Causa a $2 \mathrm{~km}$. al sur de la carretera, Trujillo. MCNG 25084, (6) (27.76-32.25mm de LE.) Puente entre quebrada Arriba y Carora. MCNG 33078, (3) (18.68-29.49mm de LE) después del caserío El Corralito, Falcón. MCNG 32179, (80) (17.98-30.02mm de LE.) río Chama en puerto Chama, Zulia. MCNG 33086, (23) (23.92-39.72mm de LE.) vía a Barito antes de llegar a agropecuaria. MCNG 33091, (7) (24.78-28.16mm de LE.). MCNG 33281, (3) (27.17-29.85mm LE.) Préstamos a $6.4 \mathrm{~km}$. antes de llegar a Alcabala de El Vigía a Santa Bárbara, Mérida. Hyphessobrycon tuyensis: Venezuela. Holotipo: IUQ 1914, (1) (30.42mm de LE.) río Capaya, tributario del río Tuy, Miranda, $10^{\circ} 19^{\prime} 20^{\prime \prime} \mathrm{N}$ y $66^{\circ} 15^{\circ} 12^{\prime}$ " W. Paratipos: MBUCV 3661, (6) (18.05$31.95 \mathrm{~mm}$ de LE.) río Tuy, en pozo aislado del curso principal cerca de Ocumare del Tuy, Miranda. MBUCV 3665, (3) (21.87-25.32mm de LE.) río Tuy, en pozo aislado del cuerpo principal cerca de Ocumare del Tuy, Miranda. MBUCV 27964, (3) (24.17-25.58mm de LE.) Tuy cerca de puerto Turarito, Miranda.

Se fotografiaron 310 individuos sobre el lado izquierdo con una cámara digital Sony Cyber Shot w360 instalada sobre un banco de fotografía a $10 \mathrm{~cm}$ del ejemplar, se utilizó la opción macro del lente de la cámara para adquirir imágenes sin deformaciones en los márgenes, y papel milimétrico como fondo en cada foto para verificar la existencia de dicha deformación (Ruiz-Calderón \& Cipriani 2006).

Los ejemplares se procesaron siguiendo el método de las cerchas (Box Truss) (Strauss \& Bookstein 1982), que consiste en la determinación de hitos o puntos homólogos en el contorno de la forma externa de los peces, de manera que sean identificables, reproducibles en todos los ejemplares estudiados y proporcionar de esta manera información relacionada con las variaciones en la forma, no sólo en el plano longitudinal sino también en el vertical (Román-Valencia \& García-Alzate 2006). Se seleccionaron 16 puntos anatómicamente homólogos (hitos), los cuales fueron ubicados en estructuras homólogas para describir la 
CUADRO 1

Coeficiente de variación alométrica para las especies de Hyphessobrycon grupo heterorhabdus presentes en Venezuela

TABLE 1

Allometric coefficient of variation for the species of Hyphessobrycon heterorhabdus-group present in Venezuela

\begin{tabular}{|c|c|c|c|c|c|c|c|}
\hline Variable & Hito & Cercha & $\begin{array}{c}H . \\
\text { diancistrus }\end{array}$ & $\begin{array}{c}H . \\
\text { fernandezi }\end{array}$ & $\begin{array}{c}H . \\
\text { paucilepis }\end{array}$ & $\begin{array}{c}H . \\
\text { sovichthys }\end{array}$ & $\begin{array}{c}H . \\
\text { tuyensis }\end{array}$ \\
\hline $\mathrm{X} 1$ & $1-2$ & I & 0.5811 & 0.2062 & 10.7 & -0.1694 & 1.026 \\
\hline $\mathrm{X} 2$ & $1-12$ & I & 1.838 & 1.832 & 5.5 & 2.021 & 3.992 \\
\hline $\mathrm{X} 3$ & $1-13$ & I & 0.5327 & 0.1808 & 9.077 & -0.1301 & 0.7413 \\
\hline $\mathrm{X} 4$ & $2-3$ & II & 2.785 & 3.189 & 7.527 & 3.303 & 4.706 \\
\hline X5 & $2-13$ & I & 0.4359 & 0.12 & 6.917 & -0.08335 & 0.3949 \\
\hline X6 & $2-12$ & I & 3.417 & 5.571 & 8.829 & 5.807 & 7.067 \\
\hline $\mathrm{X} 7$ & $2-11$ & II & 0.4054 & 0.1148 & 5.861 & -0.06503 & 0.2745 \\
\hline $\mathrm{X} 8$ & $3-4$ & III & 3.002 & 3.645 & 6.532 & 3.519 & 5.3 \\
\hline X9 & $3-12$ & II & 0.4219 & 0.07878 & 5.144 & -0.04855 & 0.2675 \\
\hline $\mathrm{X} 10$ & $3-11$ & II & 2.733 & 2.65 & 6.254 & 2.93 & 4.63 \\
\hline $\mathrm{X} 11$ & $3-10$ & III & 0.3749 & 0.07952 & 4.516 & -0.03512 & 0.1966 \\
\hline $\mathrm{X} 12$ & $4-5$ & IV & 2.559 & 2.47 & 5.423 & 2.638 & 3.852 \\
\hline $\mathrm{X} 13$ & $4-11$ & III & 0.3716 & 0.0744 & 4.404 & -0.03049 & 0.1942 \\
\hline $\mathrm{X} 14$ & $4-10$ & III & 2.053 & 1.968 & 4.673 & 2.171 & 3.009 \\
\hline $\mathrm{X} 15$ & $4-9$ & IV & 0.3832 & 0.08232 & 4.539 & -0.03257 & 0.1939 \\
\hline $\mathrm{X} 16$ & $5-6$ & V & 1.823 & 1.653 & 4.309 & 1.835 & 2.959 \\
\hline $\mathrm{X} 17$ & $5-10$ & IV & 0.388 & 0.1004 & 4.9 & -0.04142 & 0.2347 \\
\hline X18 & $5-9$ & V & 1.91 & 1.666 & 4.761 & 1.801 & 3.098 \\
\hline X19 & $5-8$ & V & 0.4284 & 0.109 & 5.939 & -0.07442 & 0.3021 \\
\hline X20 & $6-7$ & VI & 1.765 & 1.217 & 4.165 & 1.432 & 2.851 \\
\hline $\mathrm{X} 21$ & $6-9$ & V & 0.4616 & 0.1206 & 7.444 & -0.101 & 0.3648 \\
\hline $\mathrm{X} 22$ & $6-8$ & VI & 1.72 & 1.135 & 4.779 & 1.404 & 2.656 \\
\hline $\mathrm{X} 23$ & $7-8$ & VI & 0.5018 & 0.1602 & 8.835 & -0.1317 & 0.7105 \\
\hline X24 & $8-9$ & V & 1.613 & 1.213 & 4.917 & 1.438 & 2.915 \\
\hline $\mathrm{X} 25$ & $9-10$ & IV & 0.5655 & 0.1807 & 10.41 & -0.1671 & 0.9419 \\
\hline X26 & $10-11$ & III & 1.643 & 1.305 & 4.619 & 1.534 & 3.073 \\
\hline $\mathrm{X} 27$ & $11-12$ & II & 0.5827 & 0.1959 & 10.37 & -0.1626 & 0.9535 \\
\hline $\mathrm{X} 28$ & $12-13$ & I & 1.901 & 1.842 & 5.081 & 1.992 & 4.047 \\
\hline
\end{tabular}

forma general del cuerpo (Fig. 2): 1- Hocico, 2- Supraoccipital, 3- Inicio de la aleta dorsal, 4Fin de la aleta dorsal, 5- Inicio de la aleta adiposa, 6- Extremo dorsal del pedúnculo caudal, 7- Punto de origen de los radios medios caudales, 8- Extremo ventral del pedúnculo caudal, 9Fin de la aleta anal, 10- Inicio de la aleta anal, 11- Base de la aleta pélvica, 12- Base de la aleta pectoral, 13-Sínfisis, 14- Borde anterior de la órbita ocular, 15- Borde posterior órbita ocular y 16- Borde posterior de la abertura opercular. Los 13 primeros correspondieron al tipo 1, mientras que los 3 últimos correspondieron al 2 o puntos seudo homólogos dado que fueron tomados con respecto al hito 1 .

Se registraron 28 medidas mediante el diseño de cerchas; en el programa Scion Image Beta 4.02 (Scion Corporation). Por medio de la 


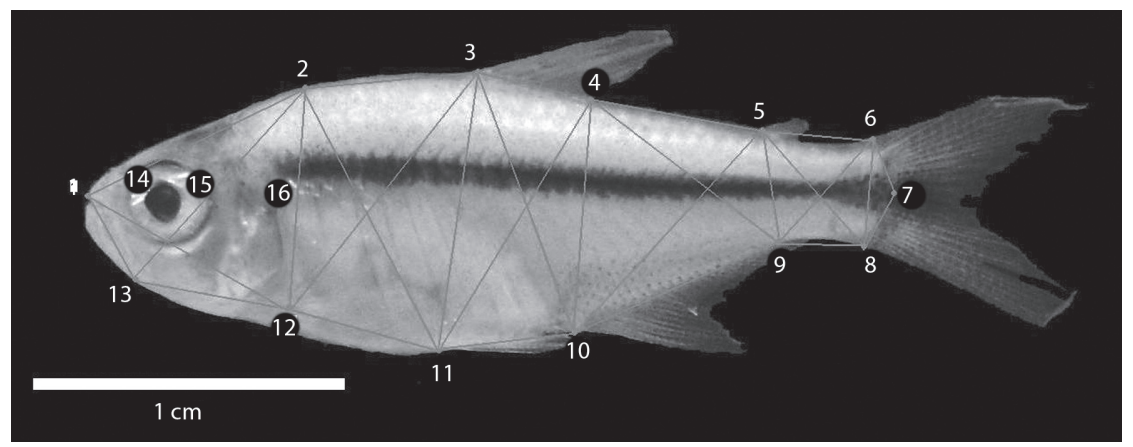

Fig. 2. Plano lateral de Hyphessobrycon paucilepis 26.77mm LE. Hitos morfológicos que indican la posición de las estructuras biológicas comparadas.

Fig. 2. Lateral view of Hyphessobrycon paucilepis $26.77 \mathrm{~mm}$ LS. Morphological landmarks that indicate the position of biological structures compared.

digitalización de los hitos sobre las imágenes de cada individuo y la determinación de las distancias entre ellos. Las coordenadas obtenidas fueron procesadas en el programa Past 1.81 (Paleontological Statistics) bajo Windows (Hammer et al. 2001), donde se llevó a cabo un Análisis de Componentes Principales (ACP), y en el que las coordenadas fueron transformadas para remover el efecto del tamaño de los ejemplares aplicando el método de Burnaby, el cual es una función que convierte a Log las medidas de distancias multivariadas y proyecta en un espacio ortogonal el primer componente principal, de manera que se maximizan las diferencias de aquellas variables que contribuyen a la separación de los grupos por su forma, independiente del tamaño (García-Alzate \& Román-Valencia 2006). Se determinó la alometría multivariada con datos transformados a logaritmo y deformaciones parciales con transformaciones de los datos en distancias de procrustes y análisis cluster con las coordenadas de la configuración consenso de cada una de las especies empleando el algoritmo UPGMA.

\section{RESULTADOS}

El análisis de componentes principales (ACP) evidenció solapamiento de formas. Las diferencias fueron explicadas mediante los interpuntos hocico-supraoccipital en el primer componente $(81.642 \%)$ y supraoccipital-inicio de la aleta pectoral en el segundo componente (11.927\%) (Fig. 3 y Cuadro 2a). El análisis de componentes principales para las especies $H$. fernandezi y $H$. sovichthys permitió visualizar que ambos taxones se distinguen en el eje $\mathrm{X}$ por la distancia entre los hitos hocico-supraoccipital y en el eje Y por la distancia entre los hitos supraoccipital-inicio de la aleta pectoral, entre el primero y segundo componente se explicó $94.06 \%$ de la variabilidad total (Fig. 4 y Cuadro 2b.). En cuanto a H. paucilepis y H. tuyensis, el ACP indicó un solapamiento de formas, la variación fue explicada mediante los interpuntos hocico-supraoccipital (CP1: 87.44\%) y supraoccipital-inicio de la aleta pectoral (CP2: 6.9519\%) (Fig. 5 y Cuadro 2c.).

Las especies de Hyphessobrycon grupo heterorhabdus presentes en Venezuela se caracterizaron por presentar un crecimiento alométrico. Se presentaron dos casos particulares: $H$. tuyensis donde se registró una isometría en la cercha I entre los interpuntos hocico-supraoccipital e $H$. paucilepis en la cual se presentó crecimiento alométrico positivo en todas sus cerchas; las demás especies se caracterizaron por aumentar la longitud y la profundidad del 


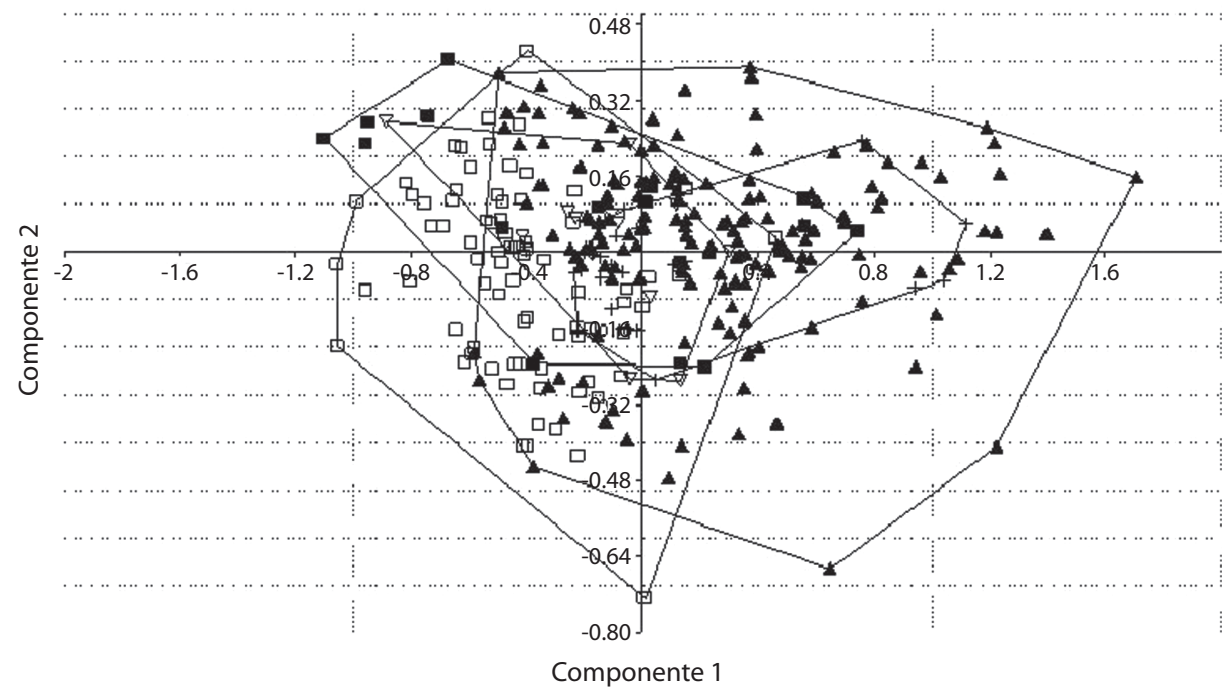

Fig. 3. Morfoespacio definido por análisis de componentes principales (Componente 1 en el eje X, componente 2 en el eje Y) para las especies de Hyphessobrycon grupo heterorhabdus de Venezuela. H. diancistrus $(+)$, H. fernandezi ( $\square)$, H. paucilepis (ם), H. sovichthys $(\boldsymbol{\Delta})$ y H. tuyensis $(\nabla)$.

Fig. 3. Morphospace defined for principal component analysis (Component 1 on $\mathrm{X}$ axis, component 2 on $\mathrm{Y}$ axis) for

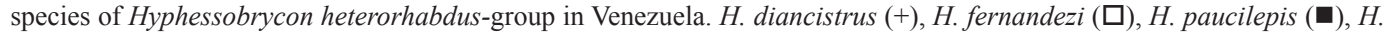
sovichthys $(\mathbf{\Delta})$ and $H$. tuyensis $(\nabla)$.

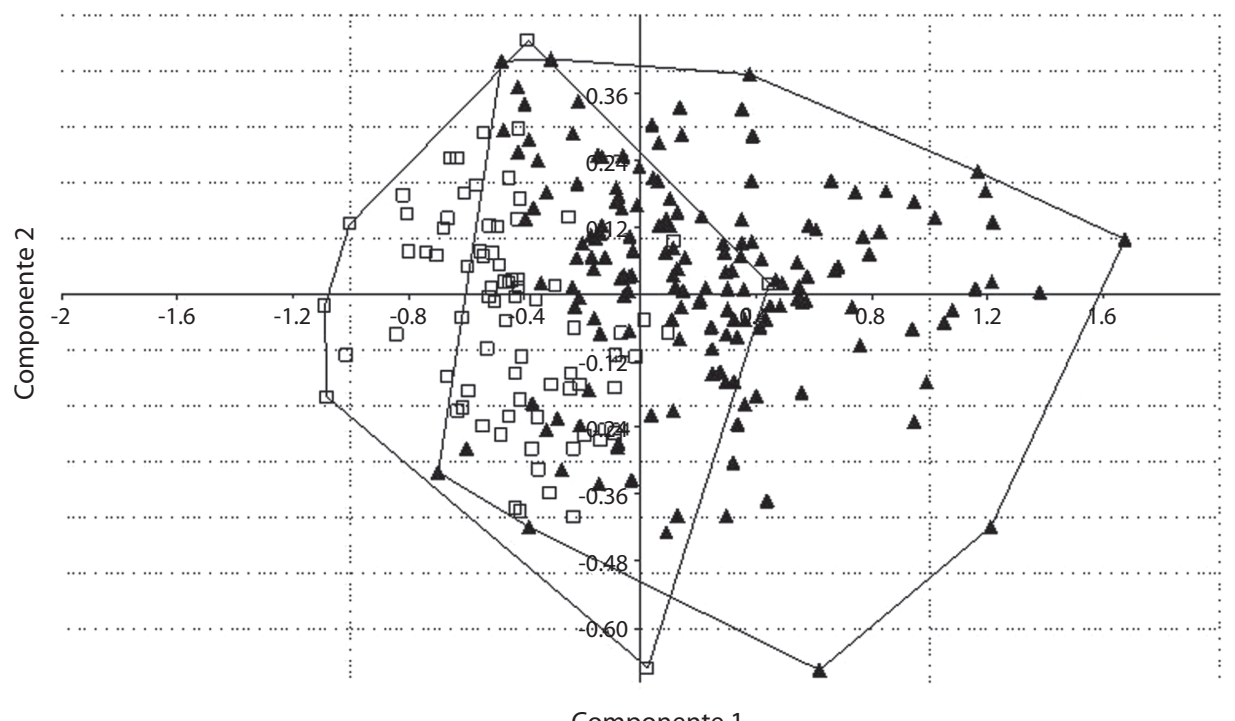

Fig. 4. Morfoespacio definido por análisis de componentes principales (Componente 1 en el eje X, componente 2 en el eje Y) para H. fernandezi $(\square)$ y H. sovichthys $(\mathbf{\Delta})$.

Fig. 4. Morphospace defined for principal component analysis (Component 1 on $\mathrm{X}$ axis, component 2 on $\mathrm{Y}$ axis) for $H$. fernandezi $(\square)$ and $H$. sovichthys $(\mathbf{\Delta})$. 
CUADRO 2

Valores propios de los componentes principales entre: a-Todas las especies. b- H. fernandezi vs. H. sovichthys. c- H. paucilepis vs. H. tuyensis

TABLE 2

Eigenvalue for principal components between: a-All species. b- H. fernandezi vs. H. sovichthys. c- H. paucilepis $v s$. H. tuyensis

\begin{tabular}{clll} 
& \multicolumn{1}{c}{$\mathrm{a}$} & \multicolumn{1}{c}{$\mathrm{b}$} & \multicolumn{1}{c}{$\mathrm{c}$} \\
$\mathrm{CP}$ & $\begin{array}{c}\text { Eigenvalue } \\
\text { (Fig. 3) }\end{array}$ & $\begin{array}{c}\text { Eigenvalue } \\
\text { (Fig. 4) }\end{array}$ & $\begin{array}{l}\text { Eigenvalue } \\
\text { (Fig. 5) }\end{array}$ \\
\hline 1 & 0.241033 & 81.78 & 0.209981 \\
2 & 0.0352117 & 12.286 & 0.0166934 \\
3 & 0.00759775 & 2.526 & 0.00704535 \\
4 & 0.00311824 & 1.0975 & 0.00184778 \\
5 & 0.00277228 & 0.45924 & 0.00149146 \\
6 & 0.00124833 & 0.43712 & 0.000861088 \\
7 & 0.00080938 & 0.26905 & 0.000492422 \\
8 & 0.00062484 & 0.22831 & 0.000354901 \\
9 & 0.000510262 & 0.17578 & 0.00029493 \\
10 & 0.000307664 & 0.11046 & 0.000219949 \\
11 & 0.000283061 & 0.085593 & 0.000165505 \\
12 & 0.000244285 & 0.078438 & 0.00013948 \\
13 & 0.000225929 & 0.072709 & 0.000122053 \\
14 & 0.000170044 & 0.058741 & $8.59743 \mathrm{E}-05$ \\
15 & 0.00015648 & 0.049565 & $7.61052 \mathrm{E}-05$ \\
16 & 0.000138301 & 0.042014 & $5.97018 \mathrm{E}-05$ \\
17 & 0.00011789 & 0.038453 & $4.70797 \mathrm{E}-05$ \\
18 & $9.18876 \mathrm{E}-05$ & 0.030898 & $3.63685 \mathrm{E}-05$ \\
19 & $8.5203 \mathrm{E}-05$ & 0.028678 & $2.53825 \mathrm{E}-05$ \\
20 & $8.38072 \mathrm{E}-05$ & 0.025957 & $2.1407 \mathrm{E}-05$ \\
21 & $7.7451 \mathrm{E}-05$ & 0.023476 & $1.57276 \mathrm{E}-05$ \\
22 & $7.01189 \mathrm{E}-05$ & 0.020177 & $1.39517 \mathrm{E}-05$ \\
23 & $5.88521 \mathrm{E}-05$ & 0.016635 & $1.21041 \mathrm{E}-05$ \\
24 & $4.32016 \mathrm{E}-05$ & 0.014295 & $1.14235 \mathrm{E}-05$ \\
25 & $4.13343 \mathrm{E}-05$ & 0.011452 & $6.53722 \mathrm{E}-06$
\end{tabular}

cuerpo a medida que crecen, mientras la región cefálica se reduce con relación al tamaño corporal, excepto las especies mencionadas anteriormente dado que en el caso de $H$. tuyensis donde la cabeza no presentó este desarrollo debido al tipo de crecimiento de esta región (Cuadro 1).

La forma consenso de $H$. diancistrus indicó que esta especie se caracteriza por presentar un cuerpo alargado y poco profundo, la región predorsal (entre el supraoccipital y el inicio de la aleta dorsal) es más grande en comparación con las demás especies; se observaron desplazamientos de los hitos 13, 14 y 16, los cuales dieron origen a expansiones (Fig. 6a). Las deformaciones parciales de $H$. fernandezi describen expansiones de la región cefálica, que son resultado del desplazamiento posterior del hito 2, 7 y 16; en la región dorsal del pedúnculo caudal se presentó una expansión debido al desplazamiento anterior del hito 5 y 12 , el cual se distancio del hito 11; acercamientos de los hitos 2-3, 4-5 y 12-13 originaron contracciones (Fig. 6b). En H. sovichthys las deformaciones originaron tres expansiones, la primera de ellas en el extremo dorsal del pedúnculo caudal que surgió como respuesta al desplazamiento posterior del hito 7, la segunda en la región cefálica por el desplazamiento anterior del hito 1 y la tercera en la región de la aleta anal por el desplazamiento anterior del hito 9; el acercamiento de los hitos 4-5, 9-10 y 15-16 arrojaron contracciones (Fig. 6c). H. fernandezi y $H$. sovichthys se caracterizaron por presentar una forma corporal similar, alargada y poco profunda; estas especies se diferenciaron entre sí por el tamaño de la cabeza (cercha I) y la longitud del pedúnculo caudal (cercha V y VI) más pequeña en $H$. sovichthys, por el tamaño de la región predorsal (cercha II) y la distancia entre la base de la aleta pectoral-sínfisis (cercha I) más pequeña en $H$. fernandezi.

En la forma consenso de H. paucilepis se visualizaron deformaciones que involucraron la expansión de la parte ventral de la región cefálica debidas al desplazamiento posterior del hito 12 y anterior de los hitos 2, 14, 15 y 16 ; se observaron contracciones entre los hitos 1-2 y 11-12 (Fig. 6d). H. tuyensis se caracterizó por presentar expansiones en la parte anterior de la región cefálica que envuelven desplazamientos hacia arriba de los hitos 1,13 y 14 ; los hitos $8-9$ se acercaron dando origen a una contracción (Fig. 6e). H. paucilepis y $H$. tuyensis tienen un contorno muy similar, un poco más robusto y menos alargado en comparación con $\mathrm{H}$. fernandezi y $H$. sovichthys; las diferencias entre estas dos especies se atribuyen a la longitud del 


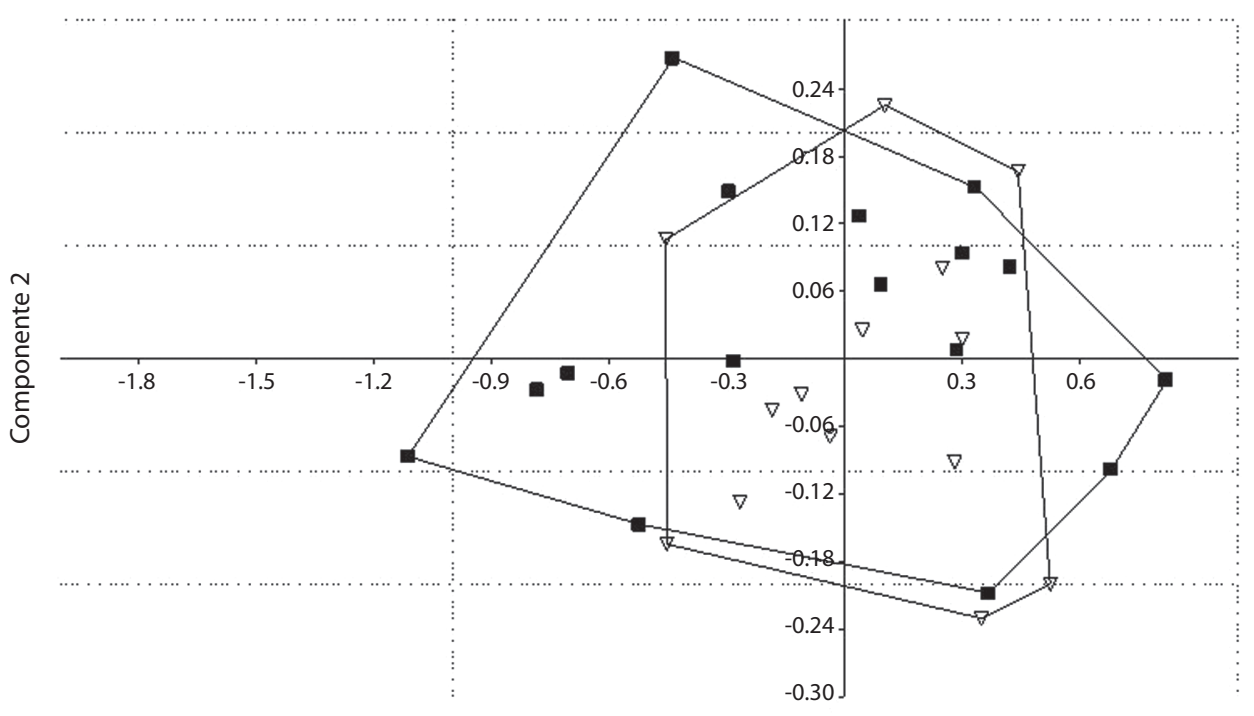

Componente 1

Fig. 5. Morfoespacio definido por análisis de componentes principales (Componente 1 en el eje X, componente 2 en el eje Y) para H. paucilepis $(\mathbf{\square})$ y H. tuyensis $(\nabla)$.

Fig. 5. Morphospace defined for principal component analysis (Component 1 on $\mathrm{X}$ axis, component 2 on $\mathrm{Y}$ axis) for $H$. paucilepis $(\mathbf{\square})$ and $H$. tuyensis $(\nabla)$.

a

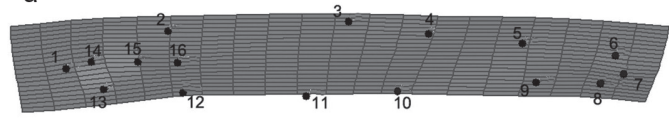

C

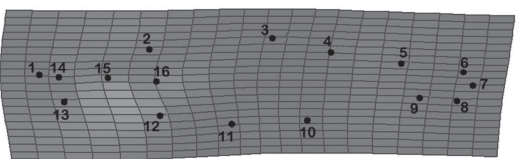

d

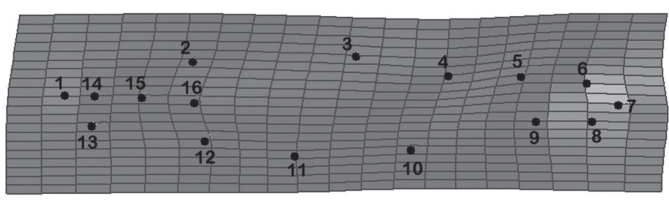

b

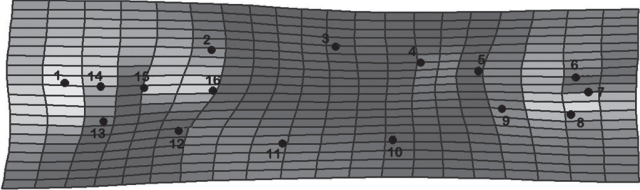

e

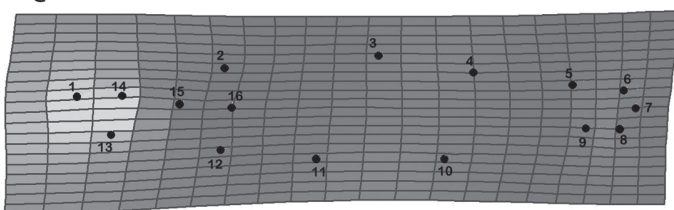

Fig. 6. Deformaciones parciales de a-Hyphessobrycon diancistrus. b- H. fernandezi. c- H. paucilepis. d- H. sovichthys. e- H. tuyensis.

Fig. 6. Partial deformations of a-Hyphessobrycon diancistrus. b- H. fernandezi. c- H. paucilepis. d- H. sovichthys. e- H. tuyensis. 
pedúnculo caudal (cercha V y VI) (más corto en $H$. tuyensis), de la base de la aleta dorsal (cercha III), distancia entre la base de la aleta pélvica y pectoral (cercha II), entre hocico y sínfisis (cercha I) (más pequeñas en $\mathrm{H}$. paucilepis). Las relaciones establecidas, con base en las comparaciones realizadas por medio de las deformaciones parciales, son corroboradas por análisis cluster, en el cual se visualiza que las especies $H$. sovichthys y $H$. fernandezi son las especies más similares; por otra parte $H$. tuyensis y H. paucilepis conformaron otro grupo que es más afín a $H$. diancistrus (Fig. 7).

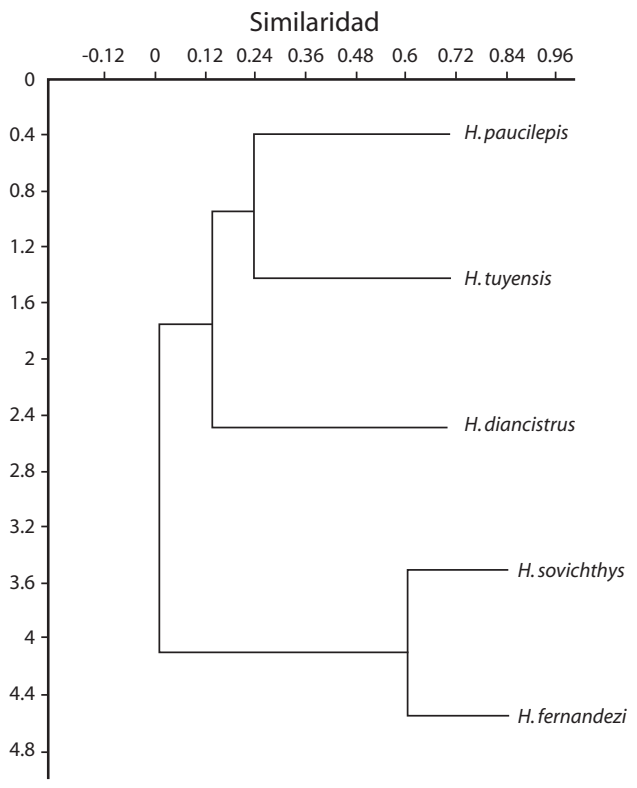

Fig. 7. Análisis Cluster por medio del algoritmo UPGMA de las especies Hyphessobrycon grupo heterorhabdus de Venezuela.

Fig. 7. Cluster analysis using the algorithm UPGMA species of Hyphessobrycon heterorhabdus-group of Venezuela.

\section{DISCUSIÓN}

El uso de cerchas permitió encontrar medidas discriminantes (hocico-supraoccipital; supraoccipital-base de la aleta pectoral), que con las tradicionales corroboraron la identidad de las cinco especies analizadas; la variación entre las especies se explica por las deformaciones en el perfil dorsal de la región cefálica y diferencias en la profundidad del cuerpo, afectadas por el crecimiento tipo alométrico de las especies.

García-Alzate et al. (2008b) realizaron un análisis de componentes principales con medidas tradicionales, y caracteres merísticos con las especies analizadas en este estudio: la mejor discriminación de los grupos fue observada sobre el primer componente $(89.93 \%)$, que describe cambios de la profundidad del cuerpo; y $H$. diancistrus fue la especie con menos semejanza morfológica con las demás. Estos resultados (García-Alzate et al.2008b) concuerdan con los registrados aquí. García-Alzate et al. (2008b) comentan que $H$. sovichthys es muy similar a $H$. fernandezi, lo que se comprueba en este estudio; así mismo plantean que $H$. paucilepis es más parecida a $H$. fernandezi, y $H$. tuyensis a $H$. sovichthys, lo cual no se ajusta con los resultados obtenidos por medio de las deformaciones parciales, al observar a $H$. fernandezi más relacionada con $H$. sovichthys, y H. tuyensis con H. paucilepis; sin embargo, las descripciones morfológicas planteadas por García-Alzate et al. (2008b) corresponden con las encontradas en la forma consenso de cada una de las especies.

García-Alzate (2009) al realizar un análisis filogenético y biogeográfico de las especies del género Hyphessobrycon grupo heterorhabdus, propone que las especies de Venezuela integran un subgrupo soportado por una sinapomorfía (región medial del mesopterigoide en contacto dorsal con el parasfenoide), más relacionado con $H$. ocasoensis, especie basal del grupo heterorhabdus, además, que $H$. fernandezi y $H$. sovichthys, H. paucilepis y H. tuyensis son especies hermanas, con base a dos sinapomorfias (escaphium no en contacto con el intercalarium y frontal sobrepuesto por encima del margen posterior del mesetmoides e insertado por debajo del parietal).

Las estrecha relación entre las especies analizadas; plantea que la semejanza morfológica puede ser producto del aislamiento geográfico reciente y de las relaciones de parentesco 
(González-Díaz et al. 2005); se comprueba dado la relación existente entre cada una de las especies y las áreas de su distribución, siendo los sistemas Perijá-Andes y Yaracuy (H. sovichthys y H. fernandezi), Tuy-Valencia y Tocuyo (H. tuyensis y H. paucilepis) áreas hermanas; lo cual permite asociar el modelo de distribución de las especies del norte de Venezuela (GarcíaAlzate et al. 2008b), con un proceso vicariante relacionado con la separación de las áreas (García-Alzate 2009).

\section{AGRADECIMIENTOS}

Se recibió financiación de la Universidad del Quindío-Vicerrectoría de Investigaciones (proyecto 357) y programa de Biología. A las siguientes personas por el préstamo, donación o acceso a material bajo su cuidado: Janeth Muñoz y Jaime Aguirre (ICMNH), Francisco Provenzano (MBUCV), Carlos A. Lasso y Oscar Lasso-Alcalá (MHNLS), Donald Taphorn (MCNG) y Douglas Rodríguez (CPUCLA). Este trabajo se beneficio de las correcciones y sugerencias de cinco evaluadores anónimos.

\section{RESUMEN}

El género Hyphessobrycon es uno de los más numerosos y morfológicamente complejos de la familia Characidae, incluye 122 especies válidas, de las cuales 17 están en Venezuela. El objetivo del presente estudio fue realizar un análisis morfogeométrico comparado entre las especies del género Hyphessobrycon grupo heterorhabdus; 310 ejemplares fueron fotografiados y analizados mediante el método de cerchas (Box Truss), por medio de la combinación de 16 hitos homólogos y un total de 28 mediciones. El análisis de componentes principales (ACP) indicó que las diferencias entre especies están relacionadas con distancia hocico-supraoccipital (81.7\%) y supraoccipital-base aleta pectoral $(11.87 \%)$; todas las especies tienen crecimiento alométrico, excepto Hyphessobrycon tuyensis, que presento isometría en la cercha I; las deformaciones parciales describen diferencias en la forma relacionadas con la región cefálica y la profundidad del cuerpo. Se plantea que la asociación de formas está relacionada con la filogenia y biogeografía de las especies.

Palabras clave: alometría, cerchas, deformaciones parciales, asociación, pez tropical.

\section{REFERENCIAS}

Eigenmann, C.H. 1917-1921. The American Characidae. Mem. Mus. Comp. Zool. Harv. Coll. 43: 1-310.

Fernández-Yépez, A. 1972. Análisis ictiológico del complejo hidrográfico (04) "Río Yaracuy". Ministerio de Obras Públicas, Dirección de Obras Hidráulicas. Caracas.

García-Alzate, C.A. 2009. Análisis filogenético y biogeográfico de las especies de Hyphessobrycon (Characiformes: Characidae) grupo heterorhabdus. Tesis de Doctorado, Facultad de Ciencias-Instituto de Zoología Tropical, Universidad Central de Venezuela, Caracas, Venezuela.

García-Alzate, C.A., C. Román-Valencia \& D. Taphorn. 2008a. Hyphessobrycon oritoensis (Characiformes: Characidae), a new species from the Putumayo River drainage, Colombian Amazon. Zootaxa 1813: 42-50.

García-Alzate, C.A., C. Román-Valencia \& D. Taphorn. 2008b. Revision of the Hyphessobrycon heterorhabdus-group (Teleostei: Characiformes: Characidae), with description of two new species from Venezuela. Vert. Zoo. 58: 139-157.

Géry, J. 1977. Characoids of the World. THF, Neptune City, New Jersey, EEUU.

González-Díaz, A., E. Díaz-Pardo, M. Soria-Barreto \& R. Rodiles-Hernández. 2005. Análisis morfométrico de los peces del grupo labialis, género Profundulus (Cyprinodontiformes: Profundulidae), en Chiapas, México. Rev. Mex. Bio. 76: 55- 61.

González-García, M. \& D. Lopera. 2009. Análisis morfométrico de las especies de Hyphessobrycon grupo heterorhabdus en Colombia. Trabajo de grado (B.Sc.), Universidad del Quindío, Armenia, Colombia.

Hammer, Ø., D.A.T. Harper \& P.D. Ryan. 2001. PAST: Paleontological Statistics Software Package for Education and Data Analysis. Paleontología Electrónica 4: 9.

Román-Valencia, C. \& C.A. García-Alzate. 2006. Análisis comparativo morfogeométrico de Characidium caucanum y Characidium sp. en el alto río Cauca, Colombia. Dahlia (Rev. Asoc. Colomb. Ictiol.) 9: 21-24.

Ruiz-Calderón, R. \& R. Cipriani. 2006. Análisis morfogeométrico de Astyanax siapae. Dahlia (Rev. Asoc. Colomb. Ictiol.) 9: 63-75. 
Strauss, R.E. \& F.L. Bookstein. 1982. The truss: body form reconstructions in morphometrics. Syst. Zool. 31: 113-135.

Schultz, L.P. 1944. The fishes of the family Characinidae from Venezuela, with descriptions of seventeen new forms. Proc. Unit. Stat. Nat. Hist. Mus. 95: 235-367.

Weitzman, S.H. 1977. A new species of Characoid fish, Hyphessobrycon diancistrus, from the río Vichada,
Orinoco river drainage, Colombia, South America (Teleostei: Characidae). Proc. Biol. Soc. Was. 90: 340-357.

Weitzman, S.H. \& L. Palmer. 1997. A new species of Hyphessobrycon (Teleostei: Characidae) from the Neblina region of Venezuela and Brazil, with comments on the putative "rosy tetra clade". Ichth. Explor. Fresh. 7: 209-242. 